\title{
Disease and subtype specific signatures enable precise diagnosis of the mucopolysaccharidoses
}

\author{
Jennifer T. Saville, $\mathrm{PhD}^{1}$, Belinda K. McDermott, $\mathrm{BSC}^{1}$, Janice M. Fletcher, $\mathrm{MD}^{1,2}$ and \\ Maria Fuller, $\mathrm{PhD}^{1,2}$
}

\begin{abstract}
Purpose: Expanding treatments for the mucopolysaccharidoses-a family of genetic disorders-place unprecedented demands for accurate, timely diagnosis because best outcomes are seen with early initiation of appropriate therapies. Here we sought to improve the diagnostic odyssey by measuring specific glycosaminoglycan fragments with terminal residues complicit with the genetic defect resulting in precise diagnosis rather than the usual first-line, ambiguous total glycosaminoglycan determinations that return poor diagnostic yield.
\end{abstract}

Methods: A derivatizing reagent was added to urine aliquots $(0.5 \mu \mathrm{mol}$ creatinine) before separation of the glycosaminoglycan fragments by liquid chromatography and quantification with electrospray ionization-tandem mass spectrometry using multiple reaction monitoring for 10 targeted fragments plus the internal standard.

Results: All 93 mucopolysaccharidosis patients were correctly identified as 1 of 10 subtypes from a total of 723 de-identified subjects-blinded to diagnosis-based on the presence of specific "signature" glycosaminoglycan fragments. Employing reference intervals calculated from 630 unaffected urines, with $99 \%$ confidence intervals, provided a laboratory test with $100 \%$ specificity and sensitivity.

Conclusion: This novel urine assay allows diagnosis of 10 mucopolysaccharidosis subtypes in a single test. The precise quantification of unique glycosaminoglycan fragments also enables longitudinal biochemical monitoring following therapeutic interventions.

Genetics in Medicine (2019) 21:753-757; https://doi.org/10.1038/s41436018-0136-z

Keywords: mucopolysaccharidosis; diagnosis; urine glycosaminoglycan

\section{INTRODUCTION}

The mucopolysaccharidoses (MPS) are a group of inherited metabolic disorders arising from a deficiency in any 1 of the 11 lysosomal hydrolases required for the degradation of glycosaminoglycans (GAGs). Incompletely degraded GAGs accumulate in the lysosomes of affected cells, leading to a cascade of intracellular abnormalities and ultimately clinical manifestations instructive of the MPS phenotype. ${ }^{1}$ In the absence of a family history, the diagnosis of MPS relies on demonstration of a reduction or absence of the specific lysosomal hydrolase in blood or cells. ${ }^{2}$ This analysis typically follows a cascade of testing beginning with an initial urine metabolic screen revealing elevated urinary GAG, usually by colorimetric detection using the 1,9-dimethylene blue reaction. ${ }^{3}$ Although this method is cheap and simple, it is a screening test that suffers from a lack of specificity and sensitivity. False positive and negative results are frequent due to interfering substances, and the natural history of the disease means that GAG levels often normalize when children finish growing. ${ }^{4}$ The next step towards a diagnosis involves GAG electrophoresis, with a resultant abnormal pattern suggestive of either MPS I, II, VI, or VII or MPS IIIA, IIIB, IIIC, or IIID. The diagnosis then relies on testing a second sample for sequential measurement in leukocytes of the enzymes responsible for each of these subtypes, until a deficiency is found. Finally, pathogenic variant testing is usually performed for confirmation.

Over the last decade a number of methods have been developed to measure broad classes of GAG, many of which have profited from the implementation and advancements in mass spectrometry platforms. ${ }^{5}$ The contemporary premise has employed various chemistries to digest/depolymerize high molecular weight GAG to smaller subunits for mass spectrometric detection. Enzymatic depolymerization has also released unique oligosaccharides that are compositionally informative for the enzyme deficiency. ${ }^{6}$ These assays are labor intensive and require expensive enzymes making them much less attractive for the routine diagnosis of MPS. As

${ }^{1}$ Genetics and Molecular Pathology, SA Pathology at Women's and Children's Hospital, North Adelaide, South Australia, Australia; ${ }^{2}$ School of Medicine, University of Adelaide, Adelaide, South Australia, Australia. Correspondence: Maria Fuller maria.fuller@adelaide.edu.au) 


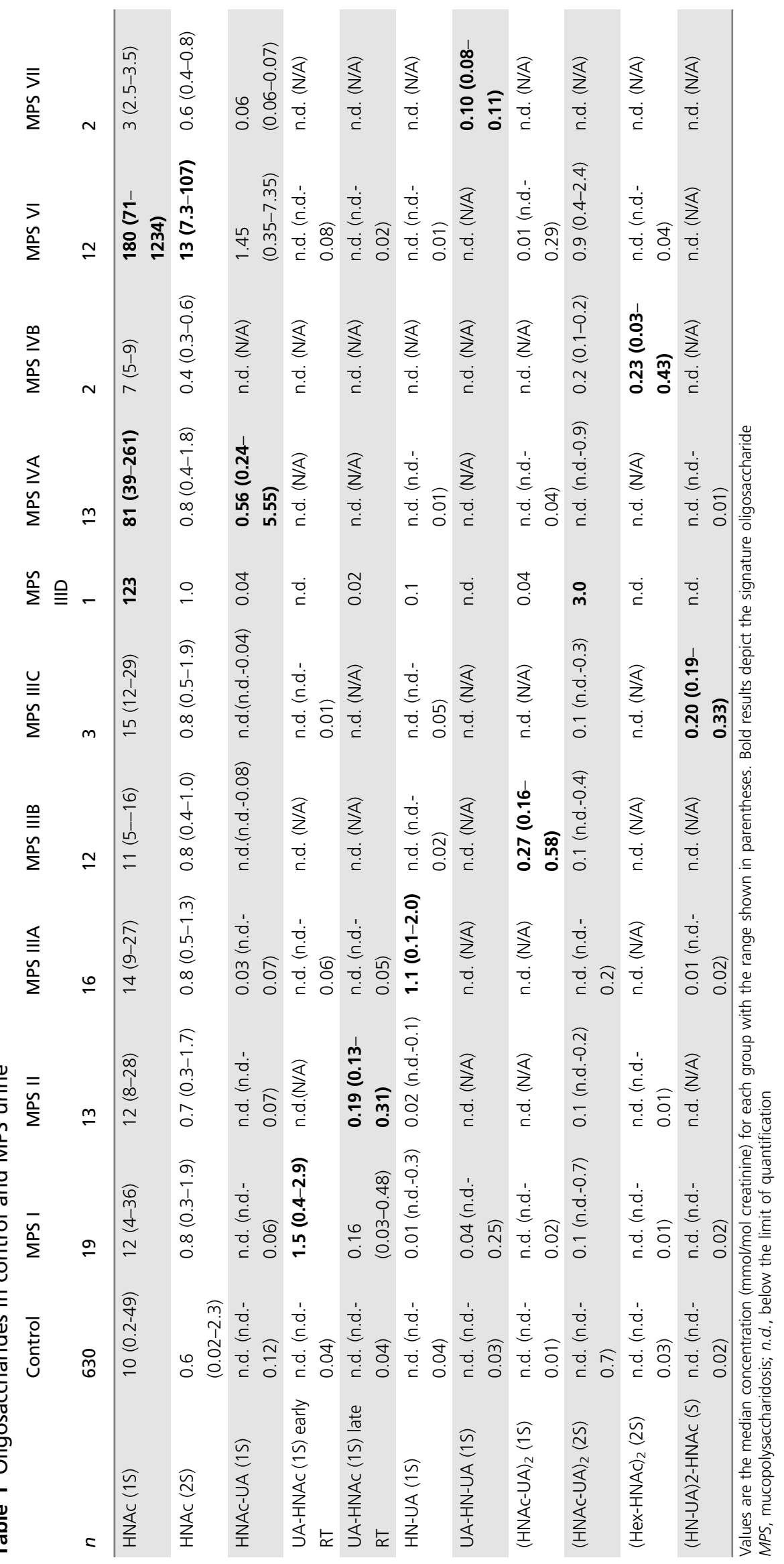


exodigestion of GAG is sequential, the deficiency of each specific enzyme results in a naturally occurring terminal residue that is the substrate for the enzyme deficiency and therefore unique for each MPS subtype. Here, we have developed a simple method that measures low molecular weight GAG fragments (oligosaccharides) with nonreducing termini in urine samples providing a diagnostic oligosaccharide signature unique for each MPS subtype, without the need for prior deconstruction of the large molecular weight GAG. This method has been applied to the routine diagnosis of 93 MPS urines showing $100 \%$ sensitivity and specificity. Sequential samples from an MPS I and VI patient in receipt of corrective therapies were also analyzed to demonstrate the usefulness of this method for biochemical monitoring.

\section{MATERIALS AND METHODS}

\section{Subjects}

This is a retrospective cohort study including urine samples that were either submitted to our Department for the diagnosis of inherited metabolic diseases or were donated by families for research purposes. The Institutional Human Ethics Committee approved their use in this study (HREC/15/ WCHN/69). Equal volumes of urine donated from individual MPS patients were used to prepare quality control (QC) materials including MPS I/II, MPS IIIABC, and MPS VI.

\section{Urinary GAG and mass spectrometry}

Total urinary GAG was determined using the method of Whitley et al. ${ }^{3}$ Preparation of urine samples for mass spectrometry of requisite GAG fragments (oligosaccharides) and validation of the methodology is detailed under Supplementary Methods and Assay Validation.

\section{Statistics}

Differences between control and MPS subtypes were analyzed for significance with a Mann-Whitney $U$ test, correlation of urinary GAG and oligosaccharides was determined by Pearson correlation coefficient, and reference intervals were calculated with a nonparametric $99 \%$ upper limit, performed with SPSS 15.0 for Windows statistical software and MedCalc ${ }^{\circledast}$ statistical software (Ostend, Belgium).

\section{RESULTS}

Identification of signature oligosaccharides for each MPS subtype

Specific oligosaccharides for each MPS subtype were determined, based on a characteristic nonreducing end, which was the substrate for the enzyme deficiency (detailed mass spectrometry can be found in Table S1). For MPS I and II, a sulfated disaccharide (UA-HNAc [1S]) was identified, and a trisaccharide (UA-HNAc-UA [1S]) was identified in both I and VII. The characteristic fragment in MPS IIIA was a sulfated disaccharide (HN-UA [1S]), in IIIB a sulfated tetrasaccharide ([HNAc-UA $\left.]_{2}[1 \mathrm{~S}]\right)$, and in IIIC a disulfated pentasaccharide ([HN-UA $\left.]_{2}-\mathrm{HNAc}[2 \mathrm{~S}]\right)$. For MPS IIID, IVA, and VI both a sulfated monosaccharide (HNAc [1S]) and a disulfated tetrasaccharide ([HNAc-UA $\left.]_{2}[2 \mathrm{~S}]\right)$ were identified with a disulfated species (HNAc [2S]) unique for MPS VI. A sulfated disaccharide (HNAc-UA [1S]) was also identified in both MPS IVA and VI, which enabled differentiation of IVA from IIID. A disulfated tetrasaccharide ([Hex-HNAc $]_{2}[2 \mathrm{~S}]$ ) with a nonreducing hexose (presumably galactose) defined MPS IVB. Chromatographic separation was important for not only eliminating urinary salt and excess derivatizing reagent, but also enabling definitive differentiation of the subtypes. For UA-HNAc (1S) present in MPS I and II urine, Fig. S1 shows that this disaccharide eluted $0.4 \mathrm{~min}$ earlier in MPS I compared with II, and similarly (HNAc-UA) (2S) eluted 0.4 min earlier in MPS IIID than in VI and IVA.

\section{Diagnosis of MPS in de-identified urine samples}

Table 1 depicts the concentrations of each oligosaccharide in urine samples from 630 metabolic controls (non-MPS) and 93 MPS patients. All signature oligosaccharides identified in each MPS subtype when compared with the control group yielded a Mann-Whitney $U$ of $0(p<0.01)$; of note, HNAc (1S) was present in MPS IIID, IVA, and VI. Using calculated reference ranges, all 93 MPS patients, along with the subtype, could be correctly identified from three separate experiments in which all samples were de-identified. The control cohort contained 56 urine samples from infants $<12$ months of age, with the youngest urine sample from a neonate at 2 days of age, and concentrations of the signature oligosaccharides were all within the reference intervals reported in Table 1.

\section{Longitudinal assessment of MPS I and VI following specific treatment}

To assess the applicability of these oligosaccharides as biomarkers for therapeutic efficacy, the signature oligosaccharides were measured in archived urine samples where available. Fig. 1 shows that the urinary signature oligosaccharides present in an MPS I and VI patient decreased by $99 \%$ following enzyme replacement therapy, and remained at this low level thereafter for the time periods monitored. Urinary GAG concentrations correlated with the respective oligosaccharides in both patients with Pearson correlation coefficients of 0.99 . Nine post-bone marrow transplant urine samples were available from an additional MPS I patientreturning UA-HNAc (1S) concentrations of between 0.05 and $0.3 \mathrm{mmol} / \mathrm{mol}$ creatinine. Although no pre-bone marrow transplant urine sample was available for this patient, the concentrations of disaccharide remained low and stable over the 15-year time period measured.

\section{DISCUSSION}

This method allows identification of ten MPS subtypes by measuring characteristic signature oligosaccharides with a terminal nonreducing end reflecting the block in GAG catabolism. Because the enzyme deficiency in each subtype is unique, a diagnostic oligosaccharide informed for all MPS subtypes (Table 1). There was no MPS IX urine available for inclusion but theoretically such urine would also contain a 

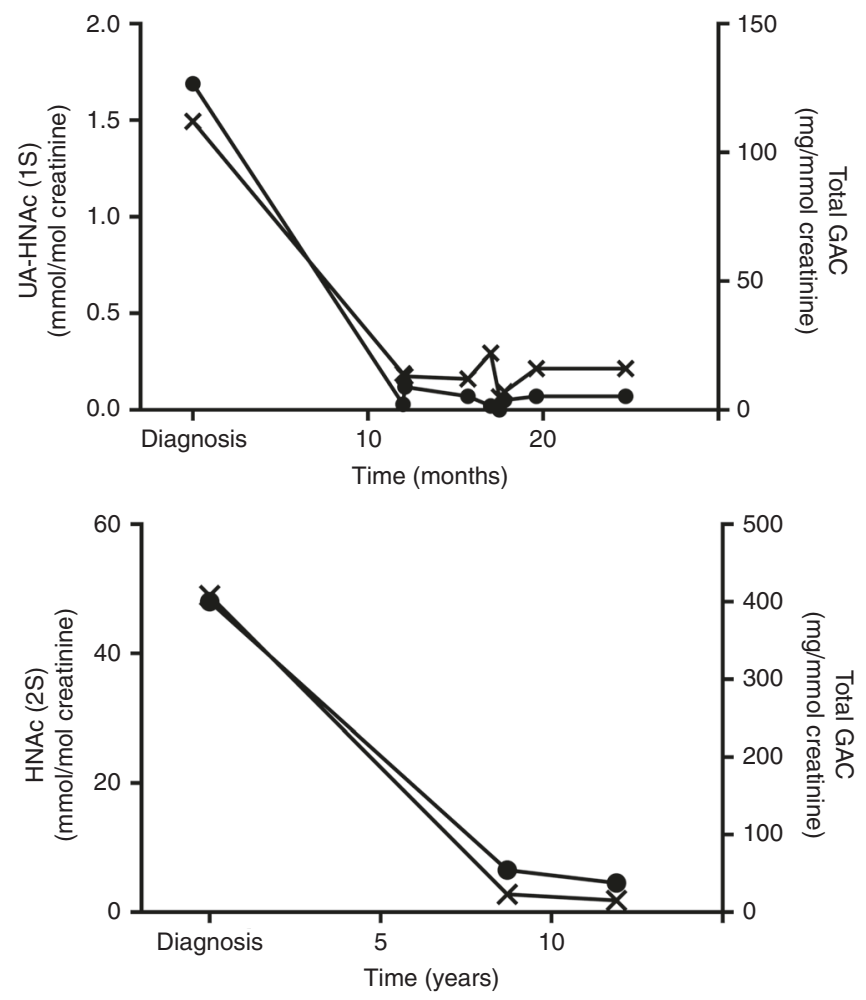

Fig. 1 Signature oligosaccharide and GAG concentrations in urine following enzyme replacement. Top and bottom panels depict an MPS I and MPS VI patient, respectively. Initial urine sample from both patients is at diagnosis, providing a single pretreatment concentration with filled circles representing the signature oligosaccharide and crosses total GAG. GAG, glycosaminoglycan; MPS, mucopolysaccharidosis.

specific GAG fragment. In addition to the signature oligosaccharides, the sulfated monosaccharide (HNAc [1S]) was present in high concentrations in MPS IIID, IVA, and VI. This is due to removal of the sulfated $N$-acetylhexosamine residues by the activity of $\beta-N$-acetylhexosaminidase on the nonreducing end GAG accumulating in these MPS subtypes, as has been previously reported. ${ }^{7}$ Unlike other urinary MPS testing methods, separate age-related reference intervals were not required, most likely because these signature oligosaccharides are small-the largest is a pentasaccharide-and hence far removed from the high molecular weight GAG that is reported to fluctuate with physiological growth and development. ${ }^{4,8}$ This highlights the potential for newborn screening as we have shown that of the three neonatal ( $<1$ week of age) urines tested, two returned oligosaccharide concentrations within the reference intervals shown in Table 1, and a third was correctly identified as MPS VI at 1 day old. This highlights the utility of the method not only for early diagnosis, which is vital for effective intervention, but also future application to newborn screening.

Derivatization of these oligosaccharides with 1-phenyl-3methyl-5-pyrazolone (PMP) ${ }^{9}$ allowed predicted electrospray ionization-tandem mass spectrometry (ESI-MS/MS) fragmentation for ease of detection. Previous studies employing PMP derivatization have either necessitated solid phase extraction C18 cartridges to desalt the samples or somewhat lengthy chromatography run times prior to MS/MS detection. ${ }^{10,11}$ Our method has capitalized on technological improvements in high-performance liquid chromatography (HPLC) employing a shorter run time of just $12 \mathrm{~min}$ that enables both salt and excess PMP to be eliminated in high aqueous (80\%) and high organic (90\%), respectively. Furthermore, chromatographic separation also benefited delineation of MPS I and II, and using the same disaccharide, UA-HNAc (1S)-UA is believed to be $\alpha$-L-iduronic acid ${ }^{12}$-in MPS II would carry a sulfate residue on the nonreducing $\alpha$-L-iduronic acid complicit with the enzyme deficiency. ${ }^{13}$ The additional sulfate would increase the $\pi-\pi$ interactions with the HPLC column, hence the UA-HNAc (2S) eluted 0.4 min later than the monosulfated counterpart (Fig.S1). Differentiation between MPS I and MPS II urine was therefore determined by RT (early and late, respectively) by measuring the same molecule by mass spectrometry. This is most likely explained by the terminal sulfate residue in the signature MPS II oligosaccharide, being labile in the ESI source. Chromatographic separation also allowed differentiation of the tetrasaccharide (HNAc-UA) $)_{2}(2 \mathrm{~S})$ in MPS IIID from the $[\mathrm{M}+2]$ isotopologue of the highly abundant HNAc (1S) in MPS IVA and VI (Fig. S1), with the monosaccharide eluting 0.4 min later from the column. One limitation of our method is the lack of commercially available standards for each of the signature oligosaccharides. As a compromise, the concentration of each oligosaccharide was determined by reference to a nonphysiological disaccharide IS ( $\triangle \mathrm{UA}$ GalNAc-4S), with the caveat that the biochemical and ionization properties of this IS may be different to that of each oligosaccharide.

Genotype/phenotype information was not available on all patients, but there are some MPS diagnoses worthy of comment to demonstrate that this method can identify MPS patients with attenuated phenotypes, that may be missed by the traditional GAG screening test. An adolescent with the attenuated (Scheie) MPS I phenotype returned a sulfated disaccharide (UA-HNAc [1S]) concentration of $1.04 \mathrm{mmol} /$ mol creatinine, clearly above the upper end of the reference range of 0.04 . In contrast, the disaccharide concentration was $2.15 \mathrm{mmol} / \mathrm{mol}$ creatinine in an infant with the classical Hurler phenotype and W402X genotype. For MPS II, an attenuated adult MPS II patient returned a concentration of 0.14 compared with $0.34 \mathrm{mmol} / \mathrm{mol}$ creatinine for a child with a rapidly progressing form of MPS II and neurological manifestations. Two teenage MPS IVA siblings, notable for normal concentrations of total urinary GAG, despite reduced enzyme activity and genetically confirmed IVA, were clearly identified with signature oligosaccharide concentrations of 0.28 and $0.24 \mathrm{mmol} / \mathrm{mol}$ creatinine, respectively (upper reference interval of 0.12). Although these preliminary findings are encouraging, further studies will be required to confirm a correlation between signature oligosaccharides and phenotype.

Figure 1 shows a precipitous drop in signature oligosaccharides for MPS I and VI patients following enzyme 
replacement therapy, consistent with previous work that demonstrated their utility as biomarkers to chart response to therapy. ${ }^{10}$ Ongoing work is directed toward confirming that the urine oligosaccharide results correlate with the patient's clinical course. ${ }^{11,14}$

This is the first, simple urine assay that is diagnostic for 10 MPS subtypes, affording not only the diagnosis of MPS but also the subtype in the same test. Importantly, diagnosis of positive cases is confirmed by the assay of only one specific enzyme, significantly reducing the cost of testing (a single enzyme instead of three or four) and time to diagnosis. Given that access to treatment relies on accurate diagnosis and that best outcomes are seen with earlier initiation of appropriate therapies, this new test has the potential to significantly aid clinical practice for MPS. With its high precision, this assay also shows promise for biochemically monitoring efficacy of current and new therapies for MPS patients.

\section{ELECTRONIC SUPPLEMENTARY MATERIAL}

The online version of this article (https://doi.org/10.1038/s41436018-0136-z) contains supplementary material, which is available to authorized users.

\section{DISCLOSURE}

The authors declare no conflicts of interest.

\section{REFERENCES}

1. Neufeld EF, Muenzer J. The mucopolysaccharidoses. In: Scriver C, Beaudet A, Sly W, Valle DThe Metabolic and Molecular Bases of Inherited Disease. New York: McGraw-Hill; 2001:3421-3452.
2. Gelb MH, Scott $C R$, Turecek F. Newborn screening for lysosomal storage diseases. Clin Chem. 2015;61:335-346.

3. Whitley $C B$, Ridnour MD, Draper $K A$, et al. Diagnostic test for mucopolysaccharidosis. I. Direct method for quantifying excessive urinary glycosaminoglycan excretion. Clin Chem. 1989;35:374-379.

4. Gray G, Claridge P, Jenkinson L, Green A. Quantitation of urinary glycosaminoglycans using dimethylene blue as a screening technique for the diagnosis of mucopolysaccharidoses: an evaluation. Ann Clin Biochem. 2007;44:360-363.

5. Kubaski F, Osago H, Mason RW, et al. Glycosaminoglycan detection methods: applications of mass spectrometry. Mol Genet Metab. 2017;120:67-77.

6. Lawrence R, Brown JR, Al-Maffraji K, et al. Disease-specific non-reducing end carbohydrate biomarkers for mucopolysaccharidoses. Nat Chem Biol. 2012;8:197-204

7. Hopwood JJ, Elliott $H$. Urinary excretion of sulphated $\mathrm{N}$ acetylhexosamines in patients with various mucopolysaccharidoses. Biochem J. 1985;229:579-586.

8. Komosinska-Vassev K, Blat D, Olczyk P, et al. Urinary glycosaminoglycan (UGAG) excretion in healthy pediatric and adolescent population. Clin Biochem. 2014:47:1341-1343.

9. Suzuki S, Okuda M, Kakehi K, Nakamura J. High-performance liquid chromatography of reducing carbohydrates as strongly ultravioletabsorbing and electrochemically sensitive 1-phenyl-3-methyl-5pyrazolone derivatives. Anal Biochem. 1989;180:351-357.

10. Fuller M, Rozaklis T, Ramsay SL, et al. Disease-specific markers for the mucopolysaccharidoses. Pediatr Res. 2004;56:733-738.

11. Nielsen TC, Rozek T, Hopwood JJ, Fuller M. Determination of urinary oligosaccharides by high-performance liquid chromatography/ electrospray ionisation tandem mass spectrometry: application to Hunter syndrome. Anal Biochem. 2010;402:113-120.

12. Fuller M, Meikle PJ, Hopwood JJ. Glycosaminoglycan degradation fragments in mucopolysaccharidosis I. Glycobiology. 2004;14:443-450.

13. Fuller $M$, Chau A, Nowak $R$, et al. A defect in exodegradative pathways provides insight into endodegradation of heparan and dermatan sulphates. Glycobiology. 2006;16:318-325.

14. Fuller M, Brooks DA, Evangelista M, et al. Prediction of neuropathology in mucopolysaccharidosis type I patients. Mol Genet Metab. 2005;84:18-24. 